\title{
A Novel Full-Parameter Ageing Modelling Approach for Capacitors based on Complex Impedance Analysis
}

\author{
Hao Liu and Guy A. E. Vandenbosch \\ ESAT-TELEMIC Research Division \\ KU Leuven \\ 3001, Leuven, Belgium
}

\author{
Tim Claeys and Davy Pissoort \\ M-Group (Mechatronics Group) \\ KU Leuven Bruges Campus \\ 8000, Brugge, Belgium
}

\begin{abstract}
Capacitors are ubiquitous in electronic devices and play a critical role in the devices' electromagnetic compatibility performance. The ageing of capacitors, due to intrinsic degradation mechanisms and external thermal and electrical stresses, has drawn a wide attention in the reliability field. The variations of the capacitance and equivalent series resistance are typically seen as representative for the ageing of capacitors, while the other parasitic parameters are usually neglected. In this study, a new full-parameter ageing modelling approach of capacitors is proposed based on complex impedance analysis. The ageing characteristics of all the proposed 7 parameters, including all parasitic parameters, are identified by carrying out an electrical overstress accelerated ageing test.
\end{abstract}

Index Terms - capacitor, accelerated ageing, complex impedance, ageing model

\section{INTRODUCTION}

CAPACITORS are widely applied in circuits for decoupling, filtering and electromagnetic interference suppression purposes. Therefore, they play a vital role in electromagnetic compatibility (EMC)) performance [1, 2]. However, after a long-time operation, degradation mechanisms can have a significant negative impact on the capacitor parameters, such as the capacitance and the equivalent series resistance (ESR). These negative impacts might result in an EMC incompliance and even a malfunction. Measuring the degradation of these parameters is a powerful method to assess the operational conditions and estimate the life expectancy in order to guarantee the reliability and safety of the electronic system [3-5]. In this method, the capacitors are usually subjected to a harsh environment, involving thermal and electrical overstress, to accelerate the ageing process. Compared with natural ageing, the accelerated ageing method enormously shortens the test period. In most of the available literature in the capacitor ageing field, the variations of ESR and capacitance values are normally considered as the criteria to evaluate the ageing condition of the capacitor [6-10]. These methods are based on the simplest equivalent circuit model, without taking into account any other parasitic parameters. The ESR and capacitance have to be characterized at a specified frequency, which largely limits the application in a wide range of frequencies. Further, the parasitic parameters' ageing

Manuscript received on 22 September 2019, in final form 23 November 2019, accepted xx Month 20yy. Corresponding author: H. Liu. characteristics, such as internal series inductance, parallel resistance, etc., cannot be examined.

In fact, the electrical behavior of non-ideal capacitors is usually described by the lumped element model [11-17] which consists of a capacitance in combination with parasitic inductances and resistances. The impedance characteristics of the capacitor are closely related to all these parameters in the lumped element model. A non-ideal distributed capacitor model was proposed in [11]. The spray ends, contacts and electrodes as well as the parasitic capacitance were taken into account to precisely show the distributions of the equivalent elements. Similarly, a model [12] that considers production specific phenomena (welding method, asymmetric and symmetric construction) was proposed based on the NCC model in [13]. It takes into account the electrode capacitance, inductance and dielectric breakdown. It represents the precise physical structure of the capacitors. In [14], a complex capacitor model considering dielectric absorption (DA) was developed. The DA capacitance and resistance are in parallel with the nominal capacitance $\mathrm{C}$, resulting in a good fit with measured impedance characteristics. In [15], a wideband and flexible modeling methodology for inductors and capacitors based on transmission-line theory is presented. With the third-order model, one can match the measured inductor or capacitor response beyond the first resonance frequency. Another transmission line circuit model was proposed by mimicking the construction of capacitors and mounting structures [16]. It accurately represents the impedance characteristics up to 1 GHz. A more complex RC ladder circuit with a voltage- 
dependent capacitance in each branch was presented in [17]. This model may be extended to $\mathrm{n}$ branches.

In terms of ageing modelling, a calendar-aging model [5] is developed using the classical Eyring's law. It is based on a heuristic capacitance loss model where the capacitance loss is a linear function with ageing time. The ageing time is an exponential function of the elevated temperature and applied voltages. Similarly, an estimated ageing model is developed based on the classical Eyring's law to quantify the charge/discharge ageing model according to voltage, temperature, and RMS current [18]. In [19], the capacitance loss is faster at the beginning of the capacitor ageing, after which it turns into a low-rate loss phenomenon. In the developed empirical model, the ageing procedure was considered as a combination of two different time constants. A multi-stress ageing model [20] was developed based on powerfunction acceleration factors. This model is more suitable for lifetime prediction of metallized polypropylene film capacitors (MPPFCs). In [21], a new lifetime prediction model was proposed where the so-called passion distribution was introduced to describe the sudden loss that occurs during accelerated ageing of metallized film capacitors. The similar research in [22] uses the gamma process deterioration model to capture the random variation and sudden loss during the ageing process.

We want to emphasize that ageing modeling methods available in literature are based on the simple impedance model where the capacitance and ESR are chosen to represent the ageing effects of capacitors. However, it is obvious that any non-ideal capacitor can be accurately described with a more complex lumped element model with parasitic parameters considered in a wide frequency range, modeling a wider range of physical phenomena. Ageing of the capacitor is translated into ageing of all these lumped components in our model. This means that our model is able to provide a more detailed set of criteria to judge whether a capacitor has reached the regime where proper functioning is not guaranteed any more, and this for many more physical phenomena than a model just based on capacitance and ESR As far as the authors know, such a complete analysis based on the analysis of the complex impedance and fully taking the additional parasitic parameters into account is not available in literature.

In this paper, first the electrical behavior of the capacitors under test is fit to a lumped parameter impedance model with 7 parameters included. Second, the impedance variation during the ageing process is monitored, leading to the general fullparameter ageing model. The impedance model for a non-ideal film capacitor is presented in Section 2. Section 3 is dedicated to observing the variations of the capacitors' impedance due to the electrical overstress accelerated ageing process. The ageing characteristics of all the parameters in the impedance model are derived. The full-parameter ageing model is presented in Section 4, where the coefficients of the ageing model are obtained. Conclusions are drawn in Section 5.

\section{IMPEDANCE MODEL OF NON-IDEAL FILM CAPACITOR}

A relatively simple impedance model is used as a starting point to fit the measurement data. The fitting - optimization algorithm is based on the nonlinear least square method in combination with the trust region algorithm [5]. In this study, the capacitors under test (CUT) are film capacitors with polypropylene (PP) and polyethylene terephthalate (PET) dielectric materials. The capacitors used are described in more detail in Table 1. All capacitors (magnitude, phase, real and imaginary parts) were measured with an electrochemical impedance spectroscopy (EIS) in a frequency band from $10 \mathrm{kHz}$ up to $5.56 \mathrm{MHz}$.

\begin{tabular}{cccc}
\multicolumn{4}{c}{ Table 1. Capacitors under test. } \\
\hline Cap NO. & $\mathrm{C} 1$ & $\mathrm{C} 2$ & $\mathrm{C} 3$ \\
\hline Dielectric & $\mathrm{PET}$ & $\mathrm{PP}$ & $\mathrm{PET}$ \\
Rated Capacitance $\left(\mathrm{C}_{\mathrm{R}}\right)$ & $470 \mathrm{nF}$ & $470 \mathrm{nF}$ & $1 \mu \mathrm{F}$ \\
Rated Voltage $\left(\mathrm{U}_{\mathrm{R}}\right)$ & $50 \mathrm{VDC}$ & $63 \mathrm{VDC}$ & $50 \mathrm{VDC}$ \\
\hline
\end{tabular}

\subsection{SIMPLE IMPEDANCE MODEL}

Figure 1 shows a simple lumped component model of the nonideal film capacitors under consideration [15]. Next to the ideal capacitor $C$, there is a parasitic inductance $L_{s}$ called the ESL (equivalent series inductance), a resistance $R_{\mathrm{s}}$ called the ESR (equivalent series resistance), which is modeling the dissipation in the dielectric materials and conductors, and a resistance $R_{P}$ called the parallel parasitic resistance, also known as leakage resistance. The latter one is representative for the imperfect insulation by the dielectric materials used within the capacitor, resulting in a very

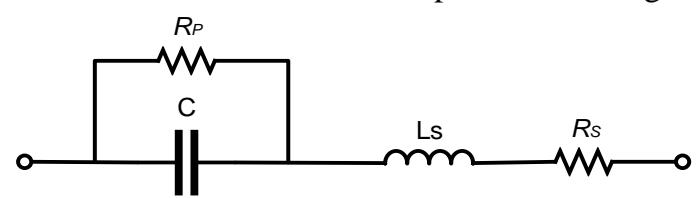

Figure 1. Simple lumped component model of non-ideal film capacitor. small current passing through the capacitor, causing a slow loss of charge in the capacitor and a dropping voltage across the capacitor. The total impedance $Z$ consists of the real part $Z^{\prime}$.and imaginary part $Z^{\prime \prime}$

$Z=Z^{\prime}+j Z^{\prime \prime}$

The real part $Z^{\prime}$ and imaginary part $Z^{\prime \prime}$ can be expressed as:

$Z^{\prime}=R_{s}+\frac{R_{p}}{1+\left(\omega C R_{p}\right)^{2}}$

$-Z^{\prime \prime}=-\omega L_{s}+\frac{\omega C R_{p}^{2}}{1+\left(\omega C R_{p}\right)^{2}}$

where $\omega$ is the angular frequency.

The magnitude of the impedance $Z$ is:

$|Z|=\sqrt{\left(Z^{\prime}\right)^{2}+\left(Z^{\prime \prime}\right)^{2}}$

and the phase angle is:

phase $=\arctan \left(Z^{\prime \prime} / Z^{\prime}\right)$

Magnitude-frequency and phase-frequency Bode plots can be used to display the frequency-response characteristics. The capacitor shows capacitive behavior when the frequency is lower than the self-resonant frequency. At this self-resonant frequency, the impedance becomes purely resistive. At higher frequencies, the impedance becomes inductive. This frequency-dependent 
character makes the capacitor sensitive to frequency shifts. Even a small shift in frequency can result in a significant variation in the magnitude and phase of the impedance.

In the lumped element model, the impedance is the sum of the real part and imaginary part which are two separate parameters. When they have large differences in magnitude, the mismatching fitting results in the relatively small-value parameter, which is more sensitive to fitting errors, are probably unnoticeable and easy to be hidden in the Bode plot after the optimization process. In this study, both Bode plot and Nyquist diagram were used to present the frequency-response characteristics of the capacitors, where the Bode plot can display the magnitude - frequency and phase frequency characteristics and the Nyquist diagram can show the details of the real part and imaginary part.

The lumped elements in the impedance model are basically identified by fitting the measured complex parts with the

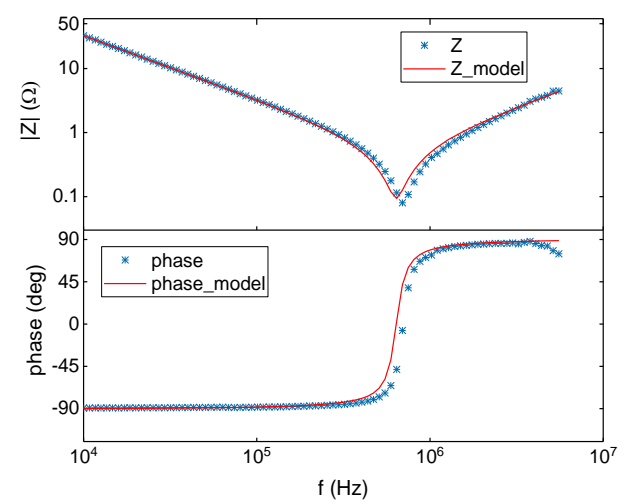

Figure 2. Bode plots of simple impedance model for $\mathrm{C} 1$.

magnitude and phase calculated according to Equation (4) and Equation (5). As an example, the Bode plot and the Nyquist diagram for $\mathrm{C} 1$ are shown in Figure 2 and Figure 3. In the Bode plot the measurements and the fitted simple impedance model seem to agree quite well, while in the Nyquist diagram large errors occur after the fitting process. The real part and imaginary part frequency-response characteristics are also presented in Figure 4.

As can be seen in Figure 4, the real part of the model is mostly constant over the frequency range and two orders of magnitude smaller than the imaginary part, which is the reason for the

Table 2. Parasitic parameters in the simple impedance model.

\begin{tabular}{cccc}
\hline \multirow{2}{*}{ Parameters } & \multicolumn{3}{c}{ Model value } \\
\cline { 2 - 4 } & $\mathrm{C} 1$ & $\mathrm{C} 2$ & $\mathrm{C} 3$ \\
\hline$C$ & $0.48 \mu \mathrm{F}$ & $1.044 \mu \mathrm{F}$ & $0.46 \mu \mathrm{F}$ \\
$L_{s}$ & $0.16 \mu \mathrm{H}$ & $0.134 \mu \mathrm{H}$ & $0.126 \mu \mathrm{H}$ \\
$R_{s}$ & $0.12 \Omega$ & $0.059 \Omega$ & $0.021 \Omega$ \\
$R_{p}$ & $0.27 \mathrm{~T} \Omega$ & $0.4 \mathrm{~T} \Omega$ & $0.27 \mathrm{~T} \Omega$ \\
R-Squared & 0.644 & 0.73 & 0.71 \\
\hline
\end{tabular}

discrepancy in the Nyquist diagram. The real part is the sum of $R_{\mathrm{s}}$ and the real part of ( $C / / R_{p}$ ), see Eq. (2). When $R_{p}$ is extremely large, $Z^{\prime}$ almost equals $R_{s}$. This happens for the capacitor considered, as can be seen in Table 2. Furthermore, the magnitude of the real part is two orders less in magnitude than the imaginary impedance values, which is the reason for the large mismatching in the Nyquist diagram. This case is a real instance to verify the aforementioned issues in the Bode plot.

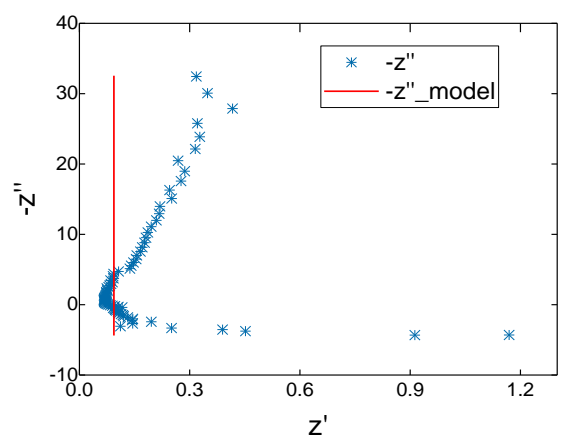

Figure 3. Nyquist diagram of simple impedance model for $\mathrm{C} 1$.

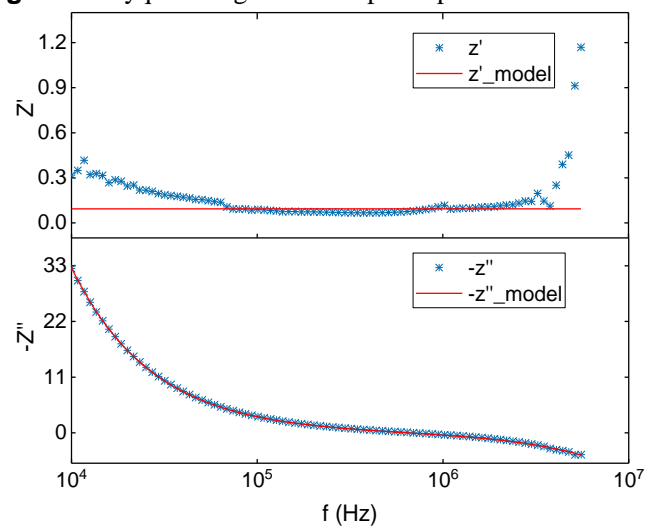

Figure 4. Real and imaginary parts vs frequency of simple impedance model for $\mathrm{C} 1$.

\subsection{COMPLEX IMPEDANCE MODEL}

In view of the discrepancy between the measurements and the simple model a more complex impedance model [14] can be employed, see Figure 5.

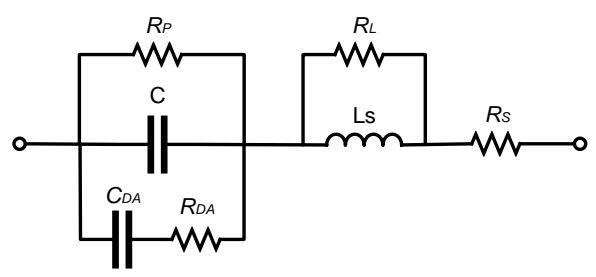

Figure 5. Complex model of non-ideal film capacitor.

The different parameters of a capacitor play a different role. The ESR models the dissipation in the dielectric materials and conductors. $R_{P}$ is representative for the imperfect insulation of the dielectrics, resulting in a very small leakage current and causing charge loss. This phenomenon can lead to a power consumption issue in micro-power applications, it can be an error source in precision analog applications, or it can result in a reliability/thermal management issue in power applications [14]. The parasitic inductance and resistance of the capacitor lead and terminal are represented by $L_{s}$ and $R_{L}$, respectively. $C_{D A}$ and $R_{D A}$ are related to the dielectric absorption [11, 14]. They model the phenomenon that after a long-time charging, when the applied voltage is removed the capacitor discharging is incomplete. There is still a measurable voltage across the capacitor. The further discharging will occur spontaneously but over a relatively long time. The phenomenon is also called dielectric relaxation or 'soakage'. This phenomenon can be problematic in precision analog circuits, but it may even pose a potentially lethal safety hazard in the context of high-voltage, 


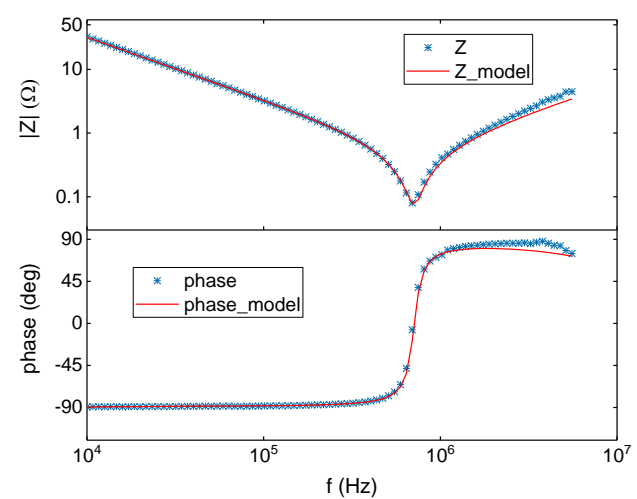

Figure 6. Bode plots of complex impedance model for $\mathrm{C} 1$.

high capacitance devices such as those used in many power factor correction or DC bus filtering applications. Note that the parasitic parameters become more important for the capacitors' performance at higher frequencies.

Considering the additional parasitic parameters, the real and imaginary parts of the complex impedance become:

$$
\begin{aligned}
& Z^{\prime}=R_{s}+\frac{\omega^{2} L_{s}^{2} R_{L}}{R_{L}^{2}+\omega^{2} L_{s}^{2}}+\frac{R_{p} \beta+\alpha \gamma}{\beta^{2}+\lambda^{2}} \\
& -Z^{\prime \prime}=\frac{\omega L_{s} R_{L}^{2}}{R_{L}^{2}+\omega^{2} L_{s}^{2}}-\frac{R_{p} \gamma+\alpha \beta}{\beta^{2}+\lambda^{2}}
\end{aligned}
$$

where

$$
\begin{aligned}
& \alpha=\omega C_{D A} R_{D A} R_{p} \\
& \beta=1-\omega^{2} C R_{p} C_{D A} R_{D A} \\
& \gamma=\omega C_{D A} R_{p}+\omega C_{D A} R_{D A}+\omega C R_{p}
\end{aligned}
$$

Figure 6 shows the Bode plot and Figure 7 the Nyquist diagram of the complex impedance model and measurements for $\mathrm{C} 1$. The model and the measurements fit much better than in case of the simple model. Figure 8 presents the real and imaginary part frequency-response characteristics, respectively. There is still a little mismatch between the real part of the model and the measured data. In [14,15], an additional RC element was connected in parallel with the ideal capacitor, yielding a better agreement between the model and the measurement data in the Nyquist diagram. In fact, more parallel RC elements can be added to have better matching results. However, additional parasitic elements further increase the complexity of the optimization process. For this reason, the above-mentioned complex impedance model was further used in this paper to extract the ageing characteristics. Table 3 gives the values of all the components in the complex impedance

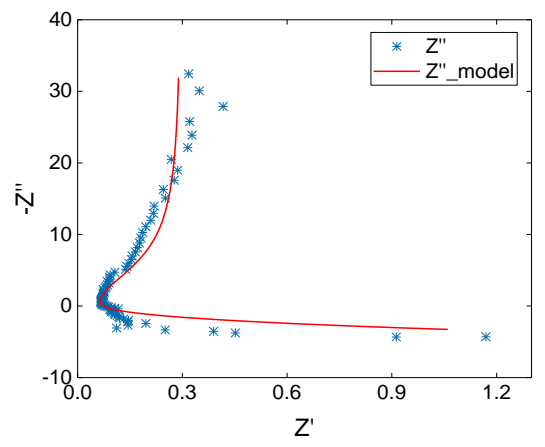

Figure 7. Nyquist diagram of complex impedance model for $\mathrm{C} 1$.

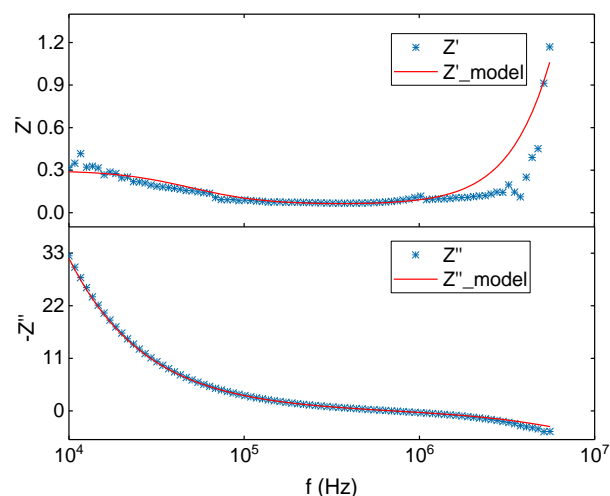

Figure 8. Real and imaginary parts vs frequency of complex impedance model for $\mathrm{C} 1$.

model. They are considered as the initial values in the accelerated ageing test.

\section{FILM CAPACITOR ACCELERATED AGEING EXPERIMENT}

Even though the time-varying capacitance and ESR values are predominantly representative of the accelerated ageing effects on the capacitors, the other parasitic parameters, such as $R_{p}$ related to the leakage current, $L_{s}$ and $R_{s}$ related to the electrode degradation, $C_{D A}$ and $R_{D A}$ related to the dielectric absorption, can have a large influence on the deterioration of the capacitor. In order to study the ageing effects in these capacitors, they were subjected to DC electrical overstress in an accelerated ageing test and the parasitic parameters in the complex impedance model introduced in Section 2 were observed as a function of time.

Table 3. Parasitic parameters in complex impedance model.

\begin{tabular}{cccc}
\hline \multirow{2}{*}{ Parameters } & \multicolumn{3}{c}{ Model value } \\
\cline { 2 - 4 }$C$ & $0.483 \mu \mathrm{F} 1$ & $0.96 \mu \mathrm{F}$ & $0.478 \mu \mathrm{F}$ \\
$L_{S}$ & $0.102 \mu \mathrm{H}$ & $0.147 \mu \mathrm{H}$ & $0.114 \mu \mathrm{H}$ \\
$R_{S}$ & $0.045 \Omega$ & $0.061 \Omega$ & $0.037 \Omega$ \\
$R_{P}$ & $0.27 \mathrm{~T} \Omega$ & $0.4 \mathrm{~T} \Omega$ & $0.27 \mathrm{~T} \Omega$ \\
$R_{L}$ & $16.63 \Omega$ & $20.01 \Omega$ & $16.17 \Omega$ \\
$C_{D A}$ & $18.2 \mathrm{nF}$ & $16.5 \mathrm{nF}$ & $9.17 \mathrm{nF}$ \\
$R_{D A}$ & $118.1 \Omega$ & $399.2 \Omega$ & $79.58 \Omega$ \\
R-Squared & 0.873 & 0.895 & 0.917 \\
\hline
\end{tabular}

\subsection{EXPERIMENTAL METHODOLOGY}

In this study, the ambient temperature was kept at $23{ }^{\circ} \mathrm{C}$. The choice of the applied voltage is crucial since an over-high voltage can result in abnormal ageing effects and even totally damage the capacitors, while a relatively low voltage leads to unnoticeable degradation of the capacitors, making the ageing test unreasonably long. Therefore, the applied voltages were chosen as $1.1 U_{R}, 1.2 U_{R}$ and $1.4 U_{R}$. The chosen 3 voltages were simultaneously applied on 3 sets of CUTs, respectively. In view of the statistical post-processing, for each voltage a set of 8 capacitors was used, making a total of 24 CUTs. The total ageing time was 1000h (about 6 weeks). During these 6 weeks the impedances of all capacitors (magnitude, phase, real and imaginary parts) were measured periodically with an 
electrochemical impedance spectroscopy (EIS) set-up in a frequency band from $10 \mathrm{kHz}$ up to $5.56 \mathrm{MHz}$. The tests used a peak-to-peak amplitude of $10 \mathrm{mV}$. The measurement result of every CUT was the average of 6 measurements in order to reduce measurement errors. To be more specific, the CUTs were exposed to the electrical stress for about 1 day, and then fully discharged prior to the measurement, in this way protecting the EIS from the high voltage. After the measurement, the voltages were applied to the CUTs again for the next 1-day period.

\subsection{EXPERIMENTAL RESULTS}

Figure 9 shows the measured impedance magnitude and phase angle variation during the accelerated ageing at $1.4 U_{R}$ for one specific $\mathrm{C} 1$ capacitor. At relatively low frequencies $(10 \mathrm{kHz}-$ $500 \mathrm{kHz}$ ) the impedance barely changes. At higher frequencies the impedance shows a gradual increase, especially around the resonant point where it noticeable ascends as seen in the zoomed figure. This increasing trend is mainly the result of the increase of $R_{s}$ in the complex model. The phase angle presents an ascending or descending shift for frequencies lower or higher than the resonant frequency, respectively. These shifts are mostly due to the variation of the capacitance value $C$ and series inductance $L_{s}$.

The Nyquist diagram in Figure 10 shows a gradual shift mainly along the real axis. Figure 11 presents the variation of the real and imaginary parts of the impedance, respectively. The real part shows an obvious increase of up to 1.8 times the initial values, corresponding to an increase of the capacitor internal resistance, while the imaginary part nearly does not vary. Note that the variation tendency of the Bode plots and the Nyquist diagram at $1.1 U_{R}$ and $1.2 U_{R}$ are virtually similar to Figure 9 and Figure 10.

\subsection{PARASITIC PARAMETER CHARACTERISTICS DURING ACCELERATED AGEING PROCESS}

To estimate the effects of the accelerated ageing process on the parasitic parameters, the variations of the parasitic parameters were identified by fitting the complex impedance model with the measurement results at each step during the accelerated ageing process. Each set of capacitors contains 8 samples with different initial values. The centered value is the mean of the fitted results for each set. The included error bar is related to the spread over the measured capacitor samples within each set. It is not related to the measurement error. The average $\bar{P}$ and the standard deviation $S D$ over the 8 capacitor samples for each capacitor type are calculated as:

$\bar{P}=\frac{1}{n} \sum_{i=1}^{n} P_{i}$

$S D=\sqrt{\sum_{i=1}^{n}\left(P_{i}-\bar{P}\right)^{2} / n-1}$

where $P_{i}$ is a sample parameter value and $n$ is the number of samples.

The results are depicted in Figure 12 and Figure 13 which are based on simple capacitor impedance model and complex model, respectively. By comparing the Figure 12 and Figure 13,
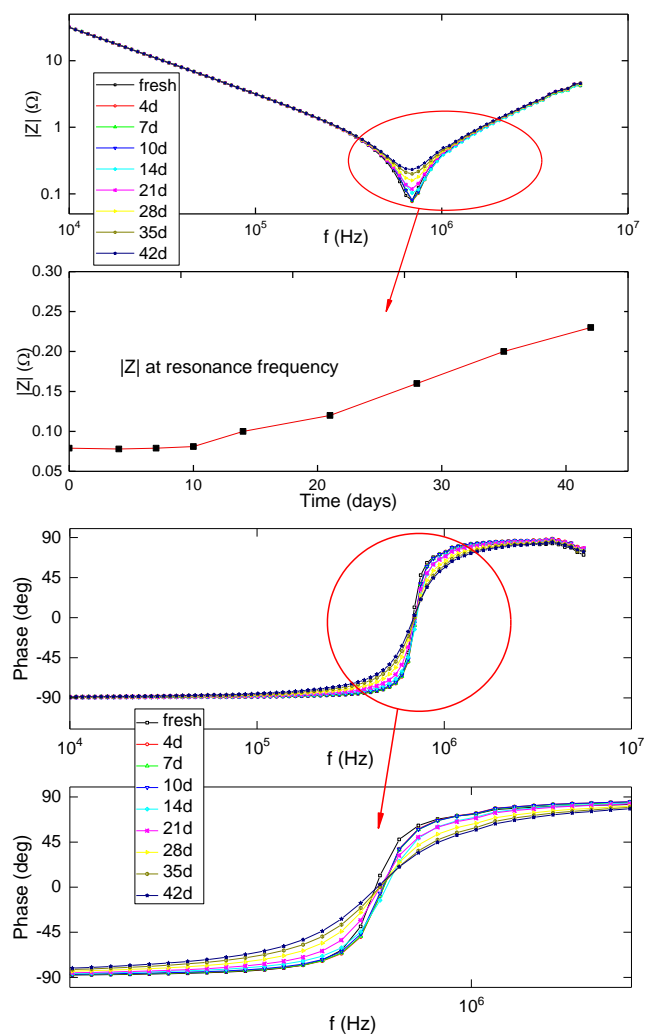

Figure 9. Impedance and phase angle variation during accelerated ageing for $\mathrm{C} 1\left(1.4 U_{R}, 23{ }^{\circ} \mathrm{C}\right)$.

the capacitance $C$, series inductance $L_{s}$ and resistance $R_{s}$ show similar variation trending along the ageing time, it means that the complexity of the capacitor impedance model will not affect the variation tendencies of the parameters.

It can be seen in Figure 12 that the parameters show limited and unnoticeable variations when the applied voltage is $1.1 U_{R}$ . In this study, the capacitors were only subjected to electrical overstress during a limited total time of $1000 \mathrm{~h}$ (about 6 weeks). Without any thermal stress or high humidity, this might result in a limited degradation in the capacitors, especially when the applied voltage is close to the rated voltage. However, when the applied voltage is increased to $1.2 U_{R}$ or $1.4 U_{R}$, the capacitance $C$ and series inductance $L_{s}$ of the CUTs show non-linear increasing and decreasing trends, respectively, which are the dominant reasons for the phase shift in Figure 9. The series resistance $R_{s}$ shows a considerable increase of up to 1.8 times the initial values which mostly leads to the ascending of the real part of the impedance. The $R_{L}$ shows a gradual decline at $1.1 U_{R}$ and $1.2 U_{R}$, and an exponential decreasing trend at $1.4 U_{R} . R_{L}$ and $L_{s}$ are closely related to the capacitor lead and terminal deterioration, whose variations can result in the shift of the magnitude and phase of the impedance. The parallel resistance $R_{p}$ barely shows any changes during the accelerated ageing process at the different applied voltages. This phenomenon might be attributed to the extremely large values which are around $0.27 \mathrm{~T} \Omega$. During the fitting procedure, $R_{p}$ had no noticeable effects on the Bode plots and Nyquist diagram by tuning the parameter. The variations of $R_{p}$ can be 


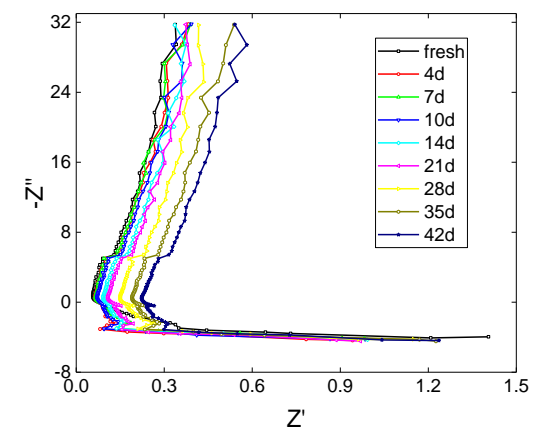

Figure 10. Nyquist diagram variation during accelerated ageing for $\mathrm{C} 1$ (1.4 $\left.U_{R}, 23{ }^{\circ} \mathrm{C}\right)$.

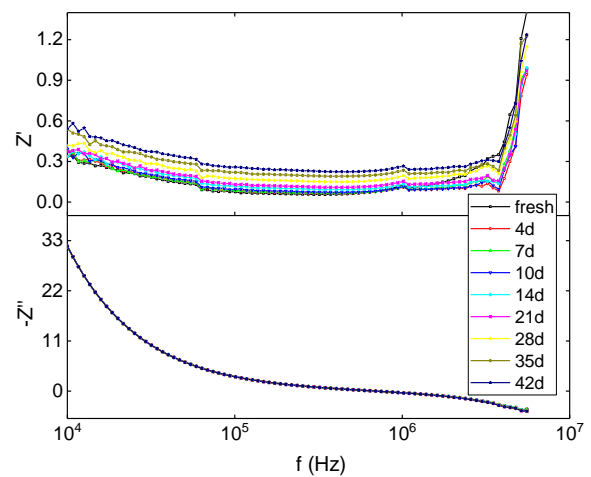

Figure 11. Real and imaginary parts variation during accelerated ageing for C1 $\left(1.4 U_{R}, 23{ }^{\circ} \mathrm{C}\right)$.

quantified by measuring the leakage current through the CUTs, which is beyond the scope of this study and will not be discussed. $C_{D A}$ and $R_{D A}$ show a gradually decreasing and increasing trend, respectively. These two parameters are directly related to the dielectric absorption, which causes a recovery voltage after the capacitors have been shorted to discharge them. Therefore, the variations of these two parameters might directly influence the capacitor performance in circuits.

\section{FULL-PARAMETER AGEING MODEL}

In the ageing model of the capacitors, the acceleration factor function is the fundamental element which can be described by
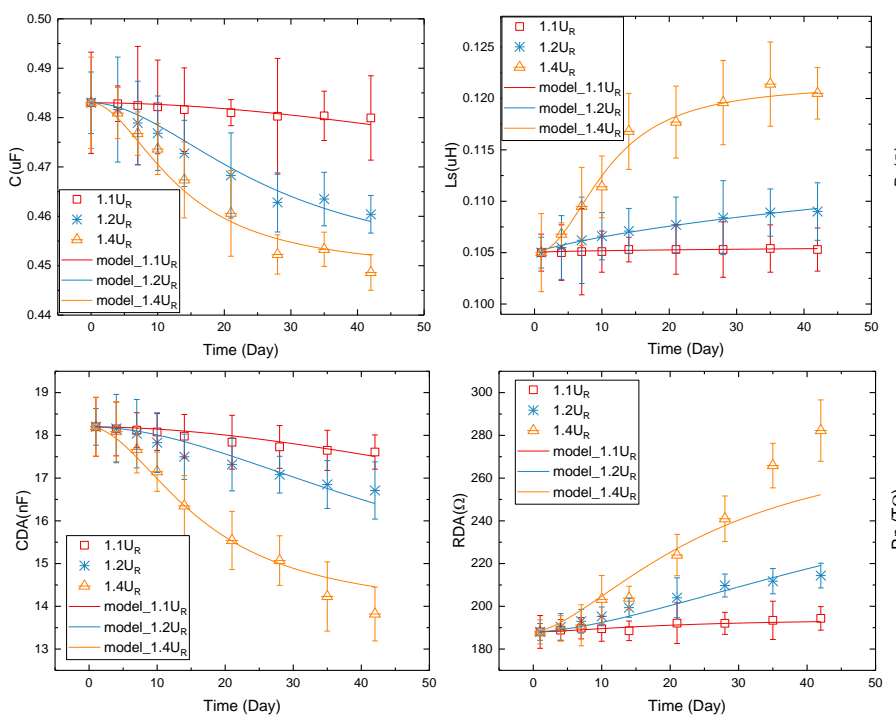
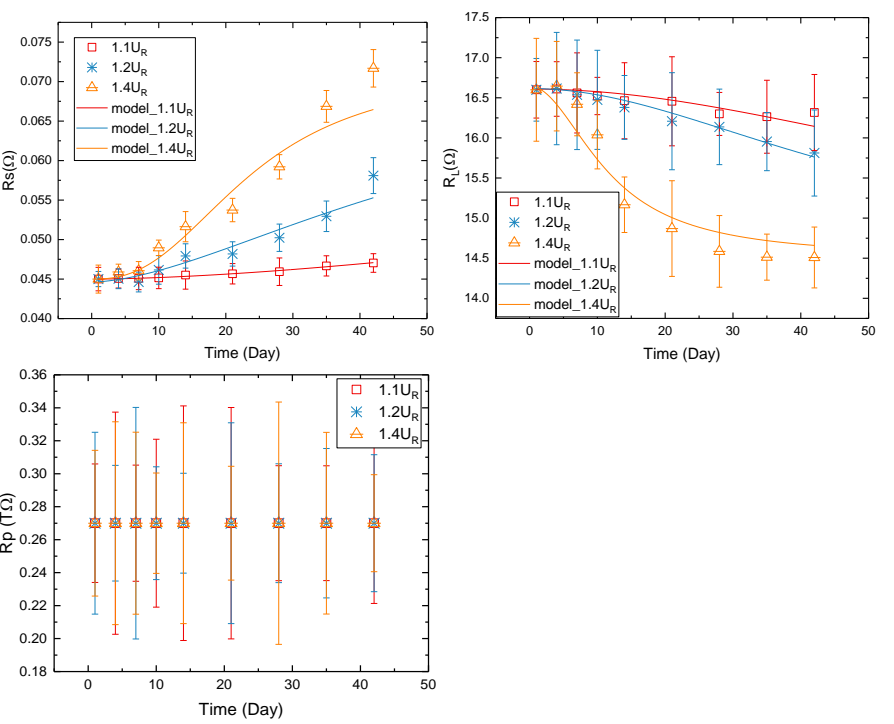

$$
P(t)=P_{0}+\gamma \operatorname{Deg}(t)
$$
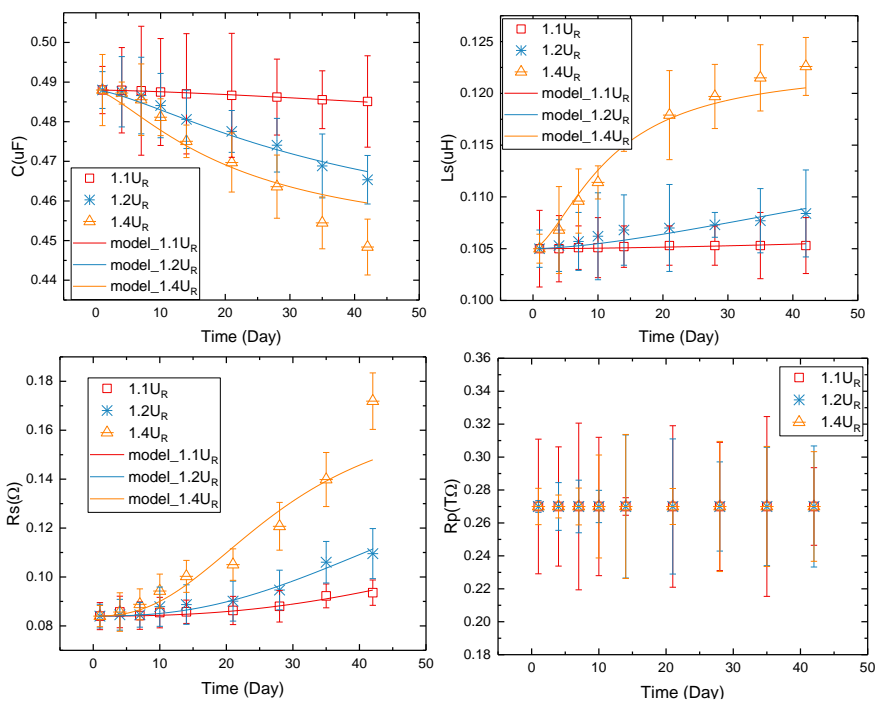
accelerated ageing test for $\mathrm{C} 1$ capacitors.

an exponential function [18, 19], a power function [20] or a linear function [5], according the ageing characteristic of the CUTs. In this paper, the capacitors' parameters show a rapid decreasing or increasing trend at the beginning, turning into a relatively slow-rate variation trend further along the ageing time.

Considering the non-linear variation characteristics of the parasitic parameters in the complex impedance model, a new general full-parameter model is proposed for ageing related to electrical overstress. In contrast to the traditional exponential model [5, 18-22], the degradation characteristic is presented as a power function

where $P(t)$ is the parameter at time $t$ during the ageing process, $P_{0}$ is related to the initial parameter, $\gamma$ is the drift parameter, and $\operatorname{Deg}(t)$ is the degradation power function, which is expressed as:

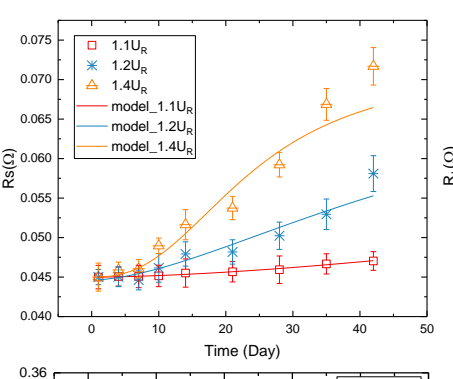

Figure 13. Measured and simulated complex model parameters during the accelerated ageing test for $\mathrm{C} 1$ capacitors. 
Table 4. Coefficients of the proposed ageing model based on simple capacitor impedance model.

\begin{tabular}{|c|c|c|c|c|c|c|c|}
\hline \multirow{2}{*}{ Capacitor } & \multirow{2}{*}{ Parameter } & \multicolumn{5}{|c|}{ Coefficients } & \multirow{2}{*}{ R-Squared } \\
\hline & & $P_{0}$ & $\gamma$ & $\lambda$ & $n$ & $v$ & \\
\hline \multirow{3}{*}{$\mathrm{C} 1$} & $C$ & $4.48 \mathrm{E}-07$ & $3.96 \mathrm{E}-08$ & 0.0027 & 8 & 1.4 & 0.92 \\
\hline & $L_{s}$ & $1.23 \mathrm{E}-07$ & $-1.76 \mathrm{E}-08$ & 0.0028 & 8 & 1.6 & 0.88 \\
\hline & $R_{s}$ & $1.72 \mathrm{E}-01$ & $-8.79 \mathrm{E}-02$ & 0.0039 & 6 & 2.5 & 0.93 \\
\hline \multirow{3}{*}{$\mathrm{C} 2$} & $C$ & $9.04 \mathrm{E}-07$ & 8.64E-08 & 0.0013 & 11 & 1.0 & 0.92 \\
\hline & $L_{s}$ & $1.17 \mathrm{E}-07$ & $2.33 \mathrm{E}-08$ & 0.0022 & 10 & 1.3 & 0.85 \\
\hline & $R_{s}$ & $1.22 \mathrm{E}-01$ & $-6.31 \mathrm{E}-02$ & 0.0046 & 8 & 1.4 & 0.92 \\
\hline \multirow{3}{*}{$\mathrm{C} 3$} & $C$ & $4.24 \mathrm{E}-07$ & $2.96 \mathrm{E}-08$ & 0.0023 & 9 & 1.1 & 0.95 \\
\hline & $L_{s}$ & $1.11 \mathrm{E}-07$ & $2.33 \mathrm{E}-08$ & 0.0011 & 10 & 0.9 & 0.91 \\
\hline & $R_{s}$ & $8.06 \mathrm{E}-02$ & $-4.86 \mathrm{E}-02$ & 0.0014 & 10 & 1.1 & 0.92 \\
\hline
\end{tabular}

Table 5. Coefficients of the proposed ageing model based on complex and simple capacitor impedance model.

\begin{tabular}{|c|c|c|c|c|c|c|c|}
\hline \multirow{2}{*}{ Capacitor } & \multirow{2}{*}{ Parameter } & \multicolumn{5}{|c|}{ Coefficients } & \multirow{2}{*}{$\begin{array}{l}\text { R-Squared } \\
\text { (complex) }\end{array}$} \\
\hline & & $P_{0}$ & $\gamma$ & $\lambda$ & $n$ & $v$ & \\
\hline \multirow{6}{*}{$\mathrm{C} 1$} & $C$ & 4.49E-07 & 3.44E-08 & 0.0051 & 9 & 1.9 & 0.94 \\
\hline & $L_{s}$ & $1.21 \mathrm{E}-07$ & $-1.64 \mathrm{E}-08$ & 0.0035 & 9 & 1.5 & 0.88 \\
\hline & $R_{s}$ & 7.17E-02 & $-2.67 \mathrm{E}-02$ & 0.0043 & 6 & 1.7 & 0.94 \\
\hline & $R_{L}$ & $1.45 \mathrm{E}+01$ & $2.14 \mathrm{E}+00$ & 0.0049 & 7 & 2.0 & 0.87 \\
\hline & $C_{D A}$ & $1.38 \mathrm{E}-08$ & 4.38E-09 & 0.0063 & 6 & 1.9 & 0.90 \\
\hline & $R_{D A}$ & $2.82 \mathrm{E}+02$ & $-9.42 \mathrm{E}+01$ & 0.0031 & 6 & 1.6 & 0.86 \\
\hline \multirow{6}{*}{$\mathrm{C} 2$} & $C$ & $8.05 \mathrm{E}-07$ & $1.55 \mathrm{E}-07$ & 0.0018 & 11 & 0.9 & 0.94 \\
\hline & $L_{s}$ & $1.02 \mathrm{E}-07$ & $5.04 \mathrm{E}-8$ & 0.0027 & 7 & 1.3 & 0.90 \\
\hline & $R_{s}$ & $9.33 \mathrm{E}-02$ & $-3.59 \mathrm{E}-02$ & 0.0030 & 8 & 1.2 & 0.94 \\
\hline & $R_{L}$ & $1.46 \mathrm{E}+01$ & $5.39 \mathrm{E}+00$ & 0.0055 & 9 & 1.5 & 0.86 \\
\hline & $C_{D A}$ & $1.19 \mathrm{E}-08$ & $4.58 \mathrm{E}-09$ & 0.0022 & 7 & 1.1 & 0.94 \\
\hline & $R_{D A}$ & $5.26 \mathrm{E}+02$ & $-1.26 \mathrm{E}+02$ & 0.0027 & 7 & 1.3 & 0.94 \\
\hline \multirow{6}{*}{$\mathrm{C} 3$} & $C$ & 4.09E-07 & $6.89 \mathrm{E}-08$ & 0.0032 & 10 & 1.2 & 0.93 \\
\hline & $L_{s}$ & 7.53E-08 & $3.87 \mathrm{E}-08$ & 0.0041 & 11 & 0.7 & 0.91 \\
\hline & $R_{s}$ & $9.19 \mathrm{E}-02$ & $-5.49 \mathrm{E}-02$ & 0.0036 & 8 & 1.4 & 0.94 \\
\hline & $R_{L}$ & $1.17 \mathrm{E}+01$ & $4.50 \mathrm{E}+00$ & 0.0036 & 10 & 1.5 & 0.94 \\
\hline & $C_{D A}$ & $6.78 \mathrm{E}-09$ & 2.39E-09 & 0.0026 & 9 & 1.3 & 0.92 \\
\hline & $R_{D A}$ & $1.77 \mathrm{E}+02$ & $-9.72 \mathrm{E}+01$ & 0.0008 & 8 & 0.8 & 0.89 \\
\hline
\end{tabular}

$\operatorname{Deg}(t)=1 / 1+\left(\lambda\left(\frac{U}{U_{R}}\right)^{n} t\right)^{v}$

where $\lambda$ is obtained from a fitting procedure, $U$ is the applied voltage, $U_{R}$ is the rated voltage of the capacitor, $n$ is the power indicator of the ratio between the applied voltage and rated voltage, and $v$ is the indicator of the power function.

The full ageing model is obtained by fitting the formula (11) with the measurements for each parameter in the complex impedance model during the accelerated ageing process. Figure 12 and Figure 13 show the comparison between the proposed ageing model and the measured parameter data of subsection 3.3 based on simple impedance model and complex impedance model, respectively. Note that the parallel resistance $R_{p}$ is not shown since it merely presents any change over the ageing time. The coefficients of the ageing model are presented in Table 4 and Table 5. The R-Squared correlation-analysis method is used [23]. It was developed to assess the Goodness-of-Fit of the fitting results. we can see that the R-Squared values in Table 5 are higher than those in Table 4, which verifies that the complex impedance model not only better describes the impedance characteristics of the capacitors, but also present better ageing characterization of the capacitors. In Table 5, all the R-Squared values of the coefficients in the model are at a good level. The proposed ageing model represents the ageing characteristics of the parameters of the capacitors with reasonable accuracy and can be seen as a powerful assistant in the prediction of the 
remaining useful lifetime up to failure of the capacitors, which can be obtained by setting $U=U_{R}$ in Equation (12) and substituting in Equation (11). In this way the natural ageing of capacitors can be obtained. When the parameters reach the failure criteria, we can say that the capacitor reaches the end of its useful lifetime. Traditional failure criteria consider the capacitor in failure when the capacitance is lower than $80 \%$ of the initial value, or the ESR value is 2 times the initial value [10]. In the proposed model, we can choose these failure criteria. However, other parameters can also be considered since they play different roles in the functions of the capacitors. E.g. if the dielectric absorption ability of the capacitor is important in circuits, the $C_{D A}$ and $R_{D A}$ should be taken into account. In our view, the optimal strategy is that the failure criteria thus depend on a preset margin related to a specific application. Furthermore, in our view, no principal indicator for failure can be decided without taking into account the device type and the precise application. However, we want to emphasize that the proposed ageing modeling approach can largely assist to predict the ageing conditions of each parameter, giving all the information needed for any type of failure criterion, related to the functionality of the capacitor under consideration.

\section{CONCLUSIONS}

The electrical performance of capacitors is determined by not only the predominant capacitance and equivalent series resistance, but also by (other) parasitic parameters, such as series inductance, and series and parallel resistance. Considering the necessity to estimate the ageing characteristics of capacitors with the parasitic parameters included, a new fullparameter ageing model for capacitors based on complex impedance analysis was proposed in this study. The procedure delivering this model goes as follows. Firstly, a complex impedance model is obtained by fitting measured impedance data. Secondly, the ageing characteristics of the parameters in this impedance model are identified by carrying out an electrical-overstress accelerated ageing process. Thirdly, the coefficients of the proposed ageing model are derived by fitting the model with the measured parameters. The new ageing model presents the ageing conditions of the capacitors' parameters with reasonable accuracy and is able to be applied to predict the remaining useful lifetime up to failure of the capacitors.

\section{REFERENCES}

[1] K. Kam et al, "EMC guideline for synchronous buck converter design," IEEE Int. Symp. on Electromagn. Compat., 2009, pp. 47-52.

[2] N. Baptistat et al, "Dynamic models of external capacitors to perform accurate EMC and ESD simulations," Int Symp. on Electromagn. Compat. (EMC EUROPE), 2017, pp. 1-6.

[3] Y. Chen et al, "Effect of interlayer air on performance of dry-type metalized film capacitor in DC, AC and pulsed applications," IEEE Trans. Dielectr. Electr. Insul., vol. 18, no. 4, pp. 1301-1306, 2011.

[4] M. Makdessi, A. Sari, and P. Venet, "Metallized polymer film capacitors ageing law based on capacitance degradation," Microelectron. Rel., vol. 54, no. 9, pp. 1823-1827, 2014.
[5] O. Bohlen, J. Kowal, and D. U. Sauer, "Ageing behaviour of electrochemical double layer capacitors: Part I. Experimental study and ageing model," J. Power Sources, vol. 172, no. 1, pp. 468-475, 2007.

[6] L. Cheng et al, "Online degradation of biaxial-orientated polypropylene film from HVDC filter capacitors," IEEE Trans. Dielectr. Electr. Insul., vol. 26, no. 1, pp. 26-33, 2019.

[7] C. S. Kulkarni et al, "Accelerated aging experiments for capacitor health monitoring and prognostics," IEEE Int. Autom. Test. Conf., 2012, 17-20.

[8] M. Ritamäki et al, "Large-area approach to evaluate DC electro-thermal ageing behavior of BOPP thin films for capacitor insulation systems," IEEE Trans. Dielectr. Electr. Insul., vol. 24, no. 2, pp. 826-836, 2017.

[9] K. Hasegawa, S. Nishizawa, and I. Omura, "ESR and capacitance monitoring of a dc-link capacitor used in a three-phase PWM inverter with a front-end diode rectifier," Microelectron. Rel., vols. 88-90, pp. 433-437, 2018

[10] Q. Qin et al, "Adaptive and robust prediction for the remaining useful life of electrolytic capacitors," Microelectron. Rel., vol. 87, pp. 64-74, 2018.

[11] H. Li et al, "Modeling of ESR in metallized film capacitors and its implication on pulse handling capability," Microelectron. Rel., vol. 55, no. 7, pp. 1046-1053, 2015.

[12] L. Kovacs et al, "Aluminium Electrolytic Capacitor Model for Capacitor Materials Structure Transformation Analysis in PWM Applications," IEEE Int. Power Electron. Motion Control Conf. (PEMC), 2018, 888-893.

[13] S. G. Parler, "Improved Spice models of aluminum electrolytic capacitors for inverter applications," IEEE Trans. Ind. Appl., vol. 39, no. 4, pp. 929-935, 2003.

[14] M. Makdessi, A. Sari, and P. Venet, "Improved Model of Metalized Film Capacitors," IEEE Trans. Dielectr. Electr. Insul., vol. 21, no. 2, pp. 582593, 2014.

[15] K. Lee, S. Mohammadi, P. K. Bhattacharya, and L. P. B. Katehi, "Compact Models Based on Transmission-Line Concept for Integrated Capacitors and Inductors," IEEE Trans. Microw. Theory Techn., vol. 54, no. 12 , pp. 4141-4148, 2006.

[16] L. D. Smith, "MLP capacitor parameters for accurate simulation model," DesignCon, 2005,pp. 1-10.

[17] D. Torregrossa et al, "Improvement of Dynamic Modeling of Supercapacitor by Residual Charge Effect Estimation," IEEE Trans. Ind. Electro., vol. 61, no. 3, pp. 1345-1354, 2014.

[18] P. Kreczanik, P. Venet, A. Hijazi, and G. Clerc, "Study of supercapacitor aging and lifetime estimation according to voltage, temperature, and RMS current," IEEE Trans. Ind. Electron., vol. 61, no. 9, pp. 4895-4902, 2014.

[19] R. Chaari, O. Briat, and J.-M. Vinassa, "Capacitance recovery analysis and modelling of supercapacitors during cycling ageing tests," Energy Convers. Manage., vol. 82, pp. 37-45, 2014.

[20] Z. Li et al, "Lifetime investigation and prediction of metallized polypropylene film capacitors," Microelectron. Rel., vol. 53, no. 12, pp. 1962-1967, 2013.

[21] Z. Li et al, "Lifetime Prediction of Metallized Film Capacitors Based on Capacitance Loss." IEEE Trans. Plasma Sci., vol. 41 no. 5, pp. 13131318, 2013.

[22] S. Shin, H. Ham, and H. Lee, "Lifetime Prediction of DC-Link Film Capacitors using a Stochastic Model Combined by Random Variable and Gamma Process." SAE Int. J. Passeng. Cars - Electron. Electr. Syst., vol. 7 no. 2, pp. 544-551, 2014.

[23] A. Colin Cameron and F. A. Windmeijer, "An R-squared measure of goodness of fit for some common nonlinear regression models," J. Econom., vol. 77, no. 2, pp. 1790-1792, 1997. 